\title{
Recomendaciones para la Selección del Material Cerámico Libre de Metal, de Acuerdo a la Ubicación de la Restauración en la Arcada
}

\author{
Recommendations for the Selection of Metal-free Ceramic Material, \\ According to the Location of the Restoration in the Arcade
}

\author{
K. J. Koushyar
}

KOUSHYAR, K. J. Recomendaciones para la selección del material cerámico libre de metal de acuerdo a la ubicación de la restauración en la arcada. Int. J. Odontostomat., 4(3):237-240, 2010.

RESUMEN: El siguiente artículo es una revisión de la literatura donde se abordan aspectos clínicos, clasificación, indicaciones de estos materiales para la elección más adecuada de la restauración. Para realizar la búsqueda bibliográfica se seleccionaron investigaciones clínicas y artículos dedicados a la difusión de resultados de investigaciones in vitro. Este trabajo servirá de guía para seleccionar una cerámica libre de metal para coronas completas con base a las características y propiedades presentadas de acuerdo a las necesidades estético-funcionales específicas de cada paciente.

PALABRAS CLAVE: libre de metal, corona, cerámica, porcelana.

\section{INTRODUCCIÓN}

En la rehabilitación protésica el área de los materiales cerámicos ha evolucionado rápidamente en los últimos años, la demanda actual de restauraciones estéticas por parte del paciente nos obliga a actualizarnos para conocer las diferentes opciones y entender cuando usar el material cerámico correcto.

En la actualidad la cerámica libre de metal ya no sólo se utiliza para fabricar coronas anteriores, puesto que muchos materiales cerámicos han demostrado el potencial para ser usados inclusive como coronas y prótesis parcial fija en posteriores (Imanishi et al., 2003). Esto se ha respaldado porque durante la masticación el esfuerzo máximo soportado en la zona de posteriores es de 500 Newtons, actualmente existen materiales que pueden resistir el doble (Griggs et al., 2007) sin embargo, las cargas en la boca son complejas y varían de persona en persona.

\section{DESARROLLO}

Al realizar una restauración libre de metal exitosa devolviendo función y estética recae una gran responsabilidad en el clínico, ya que dependerá de su habilidad decidir cuál será el diseño de la preparación (Neiva et al., 1998), la estructura de soporte dental (Burke et al., 2009), preservación periodontal (Blair et al., 2002) la selección del material apropiado de acuerdo a su composición Conrad et al., 2007), técnica de manufactura y procedimiento de cementación a seguir (Neiva et al.; Conrad et al.; Rekow et al., 2006).

Debe notarse que la resistencia de la porcelana se determina por la fase cristalina en la que sus átomos se encuentran uniformemente, mientras que la estética es determinada por la fase vítrea en la que los átomos se encuentran desordenados. La mayoría de las cerámicas dentales cuentan con esta estructura mixta, tanto matriz vítrea como cristalina MartínezRus et al., 2007). Por tanto, la traslucidez es inversamente proporcional a la resistencia, sin embargo los factores que aumentan la resistencia no dependen únicamente de la composición de las cerámicas.

Los materiales cerámicos son los mejores en imitar la apariencia natural de los dientes. Entre las 
complicaciones clínicas principales más reportadas están las fracturas de las porcelanas de recubrimiento y/o la cofia cerámica (Conrad et al.).

Tipos de cerámicas. En general la mayoría de las cerámicas dentales tienen una estructura mixta (Tabla I). En cuanto a las vitrocerámicas son materiales compuestos formados por una matriz vítrea (como el feldespato que brinda traslucidez) en la que se encuentran partículas inmersas de minerales cristalizados (disilicato de litio y leucita); al encontrarse reforzado por estos cristales, sus propiedades mecánicas (resistencia) se aumentan en relación a las porcelanas convencionales (Strappert et al., 2006; Attia et al., 2004; Zaharan et al., 2008).

Las cerámicas a base de alúmina son más resistentes que las cerámicas vítreas pero es más débil que la zirconia. Cabe mencionar que las cofias obtenidas con este grupo de materiales son menos traslúcidas, sin embargo tiene mejores características estéticas que la zirconia (Imanishi et al.; Conrad et al.; Goodacre et al., 2003; Heintze et al., 2005; Naert et al., 2005; Tsalouchou et al., 2008; Santana et al., 2009).

El zirconio es un metal duro, resistente a la corrosión, más ligero que el acero y dureza similar al cobre. Al dióxido de zirconio puro (zirconia) se le añade óxido de itrio para controlar el volumen de expansión y estabilizarla en la fase tetragonal a temperatura ambiente, obteniendo como resultado óxido de zirconio densamente sinterizado y por ende muy resistente. Actualmente, este material tiene gran demanda y amenaza con desplazar del mercado a la alúmina (Luthardt et al., 1999; Raigodski, 2004; Polack, 2006; Barnfather \& Brunton, 2007; Monicone et al., 2007; Thompson et al., 2007; Marchack et al., 2008; Zaharan et al.).

Guía de selección de la cerámica. Recopilando la información sobre las características que presentan estas distintas familias de cerámicas, he desarrollado dos cuadros que guían al clínico de manera general en la selección de los materiales disponibles de acuerdo en la zona que se encuentra ubicado el órgano $u$ órganos dentarios a rehabilitar dentro de la arcada dentaria (Fig. 1). Debemos resaltar que hay ciertos materiales que resultan ser excepciones de acuerdo a las características propias del paciente. (Parafunción, clasificación de Angle, dieta del paciente, factor socioeconómico, falta de higiene, etc.)

En la Tabla I se presenta un criterio de selección del material para rehabilitar dientes anteriores con coronas unitarias en condiciones normales.

Tabla I. Clasificación de las cerámicas por la composición su núcleo.

\begin{tabular}{|c|c|c|}
\hline Material del núcleo & Sistema & Casa comercial \\
\hline \multicolumn{3}{|l|}{ Cerámica vitrilítica } \\
\hline \multirow[t]{2}{*}{ Disilicato de lítio } & IPS e. max Press & Ivoclar Vivadent \\
\hline & IPS e. max CAD & Ivoclar Vivadent \\
\hline \multirow[t]{2}{*}{ Leucita } & IPS empress CAD & Ivoclar Vivadent \\
\hline & Finesse All-Ceramic & Dentsply \\
\hline \multirow[t]{3}{*}{ Feldespato } & VITABLOCS Mark II & Vita Zanhfabrik \\
\hline & VITA Trilux Bloc & Vita Zanhfabrik \\
\hline & VITABLOCS Ethetic Line & Vita Zanhfabrik \\
\hline \multicolumn{3}{|l|}{ Alúmina } \\
\hline \multirow[t]{4}{*}{ Óxido de aluminio } & In-Ceram Alúmina & Vita Zanhfabrik \\
\hline & In-Ceram Spinell & Vita Zanhfabrik \\
\hline & In-Ceram Zirconia & Vita Zanhfabrik \\
\hline & Procera Allceram & Nobel Biocare \\
\hline \multicolumn{3}{|l|}{ Zirconia } \\
\hline \multirow[t]{5}{*}{ Óxido } & Lava & 3M ESPE \\
\hline & Cercon & Dentsply Ceramco \\
\hline & Procera Zirconia & Nobel Biocare \\
\hline & IPS e. max ZirCAD & Ivoclar Vivadent \\
\hline & In-Ceram ZY for inLab & Vita Zanhfabrik \\
\hline
\end{tabular}


En la Fig. 2 se presenta un criterio de selección de material para rehabilitar un órgano dentario posterior con una corona unitaria en condiciones normales. Excepcionalmente se puede contar con cerámica vítrea en casos especiales en que la fuerza de masticación no sea excesiva y se busque mayor estética cuando el paciente muestre esa zona en posición dinámica como al sonreír (ejem. premolares y primer molar superiores). Debido a que en la zona de segundos molares superiores la carga oclusal es mayor, algunos prostodoncistas recomiendan rehabilitar los segundos molares con restauraciones metálicas en lugar de cerámicas.

\section{CONCLUSIÓN}

A pesar de los avances de los materiales dentales en sus cualidades ópticas, adaptación marginal,

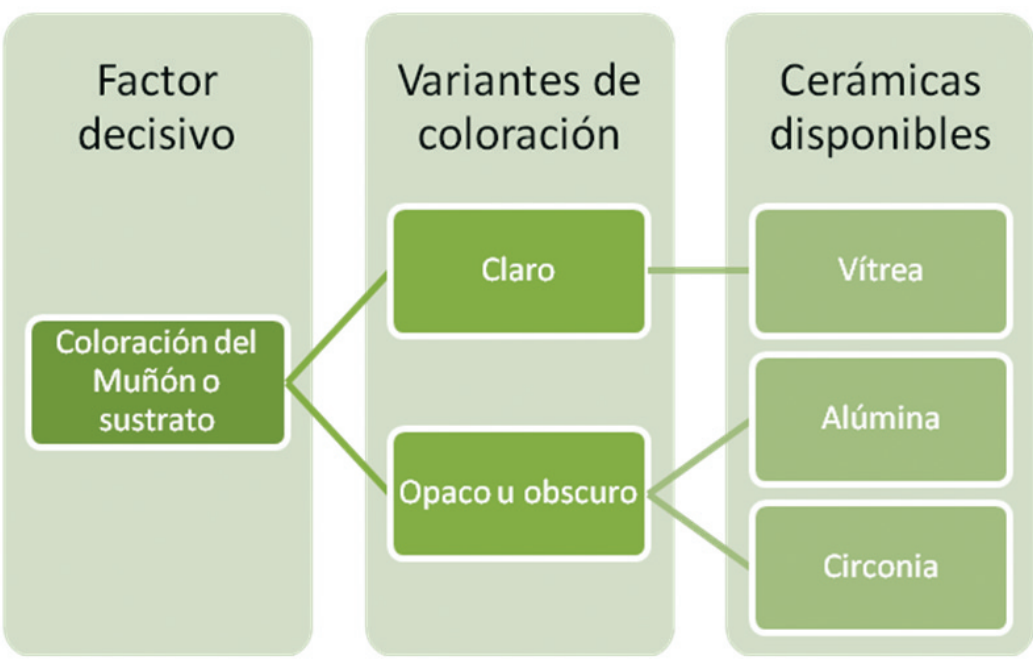

Fig. 1. Guía de selección del material para rehabilitar dientes anteriores.

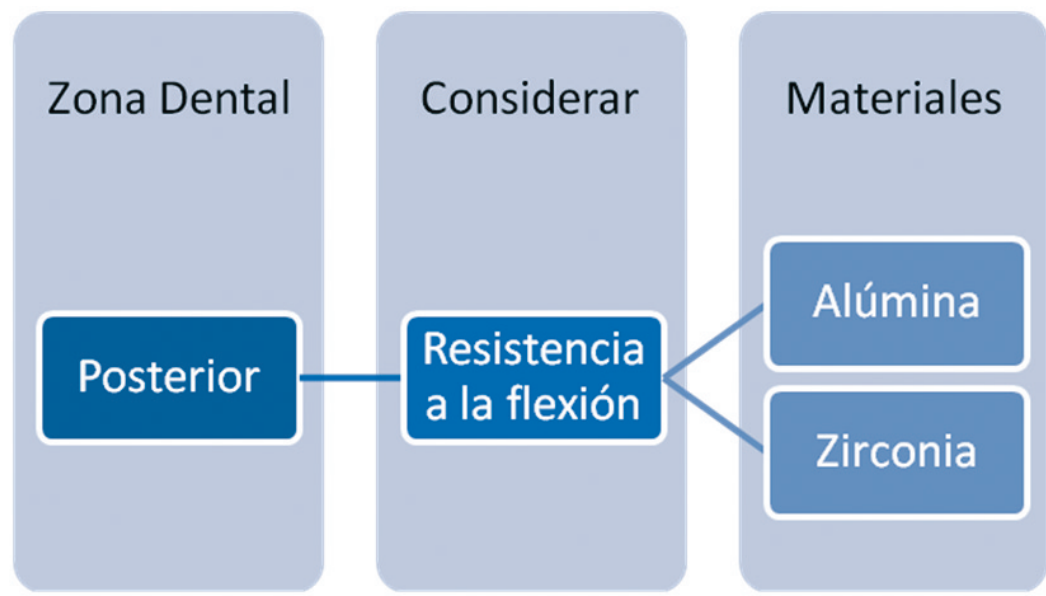

Fig. 2. Guía de selección del material para rehabilitar dientes posteriores. resistencia y biocompatibilidad, el pronóstico de la restauración estética se determina principalmente por la selección del paciente, el material de elección y la técnica empleada.

Este trabajo puede servía de guía para seleccionar el uso de una cerámica libre de metal para fabricar coronas completas tomando en cuenta las características y propiedades que presentan. Se recomienda al lector profundizar en el estudio de la literatura actual del diseño de la preparación, técnica de impresión, toma de color y cementación.

\section{AGRADECIMIENTOS}

Agradecimiento especial a la Dra. María de los Ángeles Partida MSc por haber facilitado los medios necesarios para llevar a cabo la presente investigación documental.

A la Dra. Karen García Briseño PhD Dental Materials, por su participación en la revisión.

KOUSHYAR, K. J. Recommendations for the selection of metal-free creamic material, according to the location of the restoration in the arcade. Int. J. Odontostomat., 4(3):237-240, 2010.

ABSTRACT: The following article is a review of the dental literature available, which mentions some clinical aspects, classification, indications of the materials selected for restorations. During the literature search, the articles of clinical research were selected, as well as articles dedicated to publish the results in vitro. This article is intended to be a guide in order to select a metal-free system for complete crowns based on physical properties and according to the specific esthetic and functional needs of each patient.

KEY WORDS: metal-free, fullporcelain, all-ceramic, crown, ceramic, porcelain. 


\section{REFERENCIAS BIBLIOGRÁFICAS}

Attia, A. \& Kern, M. Influence of cyclic loading and luting agents on the fracture load of two all-ceramic crown systems. J. Prosthet. Dent., 92:551-6, 2004.

Barnfather, K. D. \& Brunton, P. A. Restoration of the upper dental arch using Lava all-ceramic crown and bridgework. Br. Dent. J., 202:731-5, 2007.

Blair, F. M.; Wassell, R. W. \& Steele, J. G. Crowns and other extra-coronal restorations: preparations for full veneer crowns. Br. Dent J., 192:561-571, 2002.

Burke, F. J. T. \& Lucarotti, P. S. K. Re-intervention on crowns: What comes next? J. Dent., 37:25-30, 2009.

Conrad, H. J.; Seong, W. J. \& Pseun, I. J. Current ceramic materials and systems with clinical recommendations: a systematic review. J. Prosthet. Dent., 98:389-404, 2007.

Heintze, S. D.; Zappini, G. \& Rousson, V. Wear of ten dental restorative materials in five wear simulators-results of a round robin test. Dent. Mater., 21:304-17, 2005.

Imanishi, A.; Nakamura, T.; Ohyama, T. \& Nakamura, T. 3$D$ Finite element analysis of all-ceramic posterior crowns. J. Oral Rehabil., 30:818-22, 2003.

Goodacre, C. J.; Bernal, G.; Rungcharassaeng, K. \& Kan, J. Y. Clinical complications in fixed prosthodontics. J. Prosthet. Dent., 90(1):31-41, 2003.

Griggs, J. A. Recent advances in materials for All-ceramic restorations. Dent. Clin. North Am., 51:713-27, 2007.

Manicone, P. F.; Rossi lommetti, P. \& Raffaelli, L. An overview of zirconia ceramics: Basic properties and clinical applications. J. Dent., 35:819-26, 2007.

Marchack, B. W.; Futatsuki, Y.; Marchak, C. B. \& White, S. $\mathrm{N}$. Customization of milled zirconia copings for allceramic crowns: A clinical report. J. Prosthet. Dent., 99:169-73, 2008

Martínez-Rus, F.; Pradíes-Ramiro, G.; Suárez García, M. J. \& Rivera Gómez, B. Cerámicas dentales: clasificación y criterios de selección. RCOE, 12(4):253-63, 2007.

Naert, I.; Van der Donck, A. \& Beckers, L. Precision of fit and clinical evaluation of all-ceramics full restorations followed between 0.5 and 5 years. J. Oral Rehabil., 32:51-7, 2005.

Neiva, G.; Yaman, P.; Dennison, J. B.; Razzoog, M. E. \&
Lang, B. R. Resistance to fracture of three all-ceramic systems. J. Esthet. Dent., 10(2):60-6, 1998.

Luthardt, R. G.; Sandkuhl, O. \& Reitz, B. Zirconia-ZTP and alumina-advanced technologies for the manufacturing of single crowns. Eur. J. Prosthodont. Restor. Dent., 7:113-9, 1999.

Polack, M. A. Restoration of maxillary incisors with zirconia all-ceramic systems: a case report. Quintessence Int., 37:375-80, 2006.

Raigodski, A. J. Contemporary all-ceramic fixed partial dentures: a review. Dent. Clin. North Am., 48:531-44, 2004.

Rekow, E. D.; Harsono, M.; Janal, M.; Thompson, V. P. \& Zhang, G. Factorial analysis of variables influencing stress in all-ceramic crowns. Dent. Mater., 22:125-32, 2006.

Santana, T.; Zhang, Y.; Guess, P.; Thompson, V. P.; Rekow, E. D. \& Silva, N. R. Off-axis sliding contact reliability and failure modes of veneered alumina and zirconia. Dent. Mater., 25(7):892-8, 2009.

Strappert, C. F.; Att, W.; Gerds, T. \& Strub, J. R. Fracture resistance of different partial-coverage ceramic molar restorations: An in vitro investigation. J. Am. Dent. Assoc., 137:514-22, 2006.

Thompson, J. Y.; Stoner, B. R. \& Piascik, J. R. Ceramics for restorative dentistry: Critical aspects for fracture and fatigue resistance. Mater. Sci. Eng. C Biomim. Mater. Sens. Syst., 27:565-69, 2007.

Tsalouchou, E.; Cattell, M. J.; Knowles, J. C.; Pittayachawan, P. \& McDonald, A. Fatigue and fracture properties of yttria partially stabilized zirconia crown systems. Dent. Mater., 24:308-18, 2008.

Zaharan, M.; El-Mowafy, O.; Tam, L.; Watson, P. A. \& Finer, Y. Fracture strength and fatigue resistance of all-ceramic molar crowns manufactured with CAD/CAM technology. J. Prosthodont., 17:370-7, 2008.

Dirección para correspondencia:

K. J. Koushyar

Cuernavaca y DF México

MÉXICO

Email: kiakou@hotmail.com

Recibido : 27-08-2010

Aceptado: 26-10-2010 